\title{
A quasi-optimal design formula of a parallel-type double-mass dynamic vibration absorber based on the stability criterion
}

\author{
Toshihiko ASAMI*, Keisuke YAMADA** and Natsuki KAWAGUCHI* \\ * Department of Mechanical Engineering, University of Hyogo \\ 2167 Shosha, Himeji, Hyogo, 671-2280, Japan \\ E-mail: asami@eng.u-hyogo.ac.jp \\ ** Department of Mechanical Engineering, Kansai University \\ 3-3-35 Yamate-cho, Suita, Osaka, 564-8680, Japan
}

Received: 24 December 2020; Revised: 4 March 2021; Accepted: 20 April 2021

\begin{abstract}
In recent years, many researchers have been attempting to optimize multi-mass dynamic vibration absorbers (DVAs) to improve the performance and robustness. To date, exact design formulas have been obtained for seriestype double-mass DVAs, but for parallel-type double-mass DVAs, the optimal solutions have only been obtained in numerical form. Moreover, the expressions for calculating the numerical solutions were extremely long, making it impossible for the average user to obtain accurate design values. Another problem with the previously published expressions is that their length amplifies the rounding errors under certain calculation conditions, and sometimes makes the calculations impossible. In this paper, we propose an approximate design formula for a parallel-type double-mass DVA that is almost comparable to the exact solution in terms of accuracy. The deterioration rate of the damping performance has been confirmed as less than $0.015 \%$ in the typical use range of DVAs (mass ratio with the primary system of less than 0.2 ).
\end{abstract}

Keywords : Vibration, Optimal design, Double-mass dynamic vibration absorber, Parallel-type absorber Stability criterion, Damped primary system, Triple characteristic root, Algebraic manipulation

\section{Introduction}

A dynamic vibration absorber (DVA) is a small vibrating body attached to an object to suppress the vibrations of the object. Typical design methods for a DVA include the $H_{\infty}$ optimization criterion (Ormondroyd and Den Hartog, 1928) for minimizing the height of the resonant point of an object, the $\mathrm{H}_{2}$ optimization criterion (Crandall and Mark,1963) for minimizing the vibrational energy over the entire frequency range, and the stability maximizing criterion (Nishihara and Matsuhisa, 1997) for attenuating free vibration in the shortest time.

Research on basic single-mass DVAs has a long history (Brock, 1946; Asami et al., 2002; Asami, 2020), but recently, research on series- and parallel-type multi-mass DVAs has started to improve the performance of vibration suppression and robustness (Iwanami and Seto,1984; Kamiya et al., 1996; Yasuda and Pan, 2003; Pan and Yasuda, 2005). One of the authors attempted to optimize the double-mass DVAs based on the three optimization criteria described above and found that the simple algebraic exact solutions (Asami, 2019) for the series-type double-mass DVA can be obtained for all of the three criteria. However, for the parallel-type double-mass DVA, the solution was only available in numerical form. In addition, one of the simultaneous equations used to derive the numerical solution was extremely long, which caused the calculation error to be very large under certain conditions.

The reason for the difficulty in deriving the optimal design conditions for the parallel-type DVA is that the number of parameters to be optimized is one greater than that for the series-type DVA. To be more specific, the number of parameters to be optimized for both types of DVAs was initially five, but the final number can be reduced to four for the series-type DVA because the optimal value of one parameter becomes zero (Asami, 2017).

Therefore, an attempt was made in this study to reduce the number of parameters to be optimized from five to four for the parallel DVA. As shown in a previous paper (Asami and Yamada, 2020), the values of the optimal damping ratio 
of two DVAs arranged in parallel are very close. Thus, imposing that these damping ratios are equal, an approximate design formula for the DVA was derived. By this approximation, it was found that the vibration damping performance of a DVA was slightly degraded, but the rate of degradation was less than $0.015 \%$ in the normal use range of DVAs. The most notable advantage of this approximation is that the equation for calculating the optimal design conditions of DVAs becomes much shorter, and the error in the numerical calculation is dramatically reduced.

\section{Definition of dimensionless parameters}

Figure 1 shows an analytical model of a primary system $P$ with three types of DVAs. Letting $\omega_{1}, \omega_{2}$, and $\omega_{3}$ be the undamped natural angular frequencies of the primary system $P$, DVA- $A$, and DVA- $B$, respectively, the ratio $\left|x_{1} /\left(f / k_{1}\right)\right|$ of the amplitude of the steady-state response $x_{1}(t)$ to that of the sinusoidal input $f(t)=f_{0} \sin \omega t$ and the free-vibration response $\omega_{1} x_{1}(t) / v_{0}$ to the initial velocity $v_{0}$ can be fully represented by the following nine dimensionless parameters:

$$
\lambda=\frac{\omega}{\omega_{1}}, \tau=\omega_{1} t, \mu=\frac{m_{2}+m_{3}}{m_{1}}, \mu_{B}=\frac{m_{3}}{m_{2}}, v=\frac{\omega_{2}}{\omega_{1}}, v_{B}=\frac{\omega_{3}}{\omega_{2}}, \zeta_{1}=\frac{c_{1}}{2 m_{1} \omega_{1}}, \zeta_{2}=\frac{c_{2}}{2 m_{2} \omega_{2}}, \zeta_{3}=\frac{c_{3}}{2 m_{3} \omega_{3}},(1)
$$

where

$$
\omega_{1}=\sqrt{k_{1} / m_{1}}, \quad \omega_{2}=\sqrt{k_{2} / m_{2}}, \quad \omega_{3}=\sqrt{k_{3} / m_{3}} .
$$

It was assumed that both $\lambda$ and $\tau$ vary from zero to infinity and that a larger value of $\mu$ produces better DVA performance. When the mass ratio $\mu$ of the DVAs to the primary system and the value of the damping ratio $\zeta_{1}$ for the primary system are given, the remaining five dimensionless parameters have optimal values. Determining these optimal values is the problem of optimizing the DVA for the double-mass DVA system.

\section{Evaluation index for the stability criterion}

Although the $H_{\infty}$ and $H_{2}$ optimization criteria focus on optimizing the steady-state response of a vibratory system subjected to forced excitation, the stability criterion proposed most recently (Nishihara and Matsuhisa, 1997) is to attenuate the free-vibration response of the system in the shortest time. The evaluation index is given as

$$
\Lambda=-\max \left(\operatorname{Re}\left[s_{i}\right]\right)
$$

Here, parameter $s_{i}$ denotes the $i$-th characteristic root of the vibratory system, and the stability index $\Lambda$ represents the horizontal distance from the rightmost characteristic root to the coordinate origin, as shown in Fig. 2. Maximizing $\Lambda$ is the goal of this criterion, and its maximum value is denoted by $\Lambda_{\max }$. There is a trade-off relationship between the real parts of the different characteristic roots near the optimal condition, thus the stability is maximized when there is a repeated root with respect to the real parts. When the real parts are the same, the imaginary parts of the characteristic roots also coincide, so the stability of the system is maximized when the characteristic roots constitute a triple root in a three-degree-of-freedom (3-DOF) system (double root in a two-degree-of-freedom (2-DOF) system).

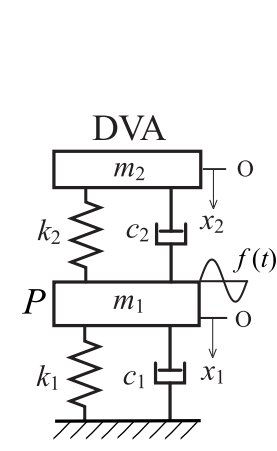

(a) Single-mass DVA

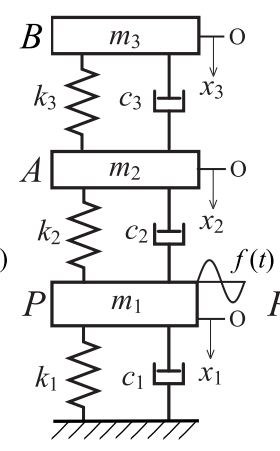

(b) Series-type doublemass DVA

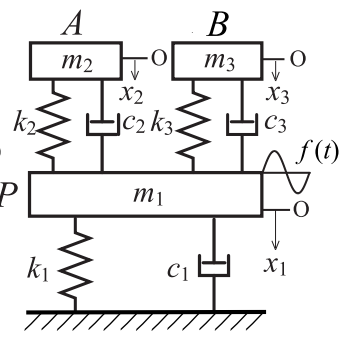

(c) Parallel-type double-mass DVA

Fig. 1 Analytical models of the vibratory system with three different DVAs. In this study, optimization of the parallel-type double-mass DVA in (c) is attempted. While the exact optimal design conditions for the series-type double-mass DVA shown in (b) have already been obtained as simple algebraic formulas, the solution for the parallel-type DVA has only been obtained in numerical form.

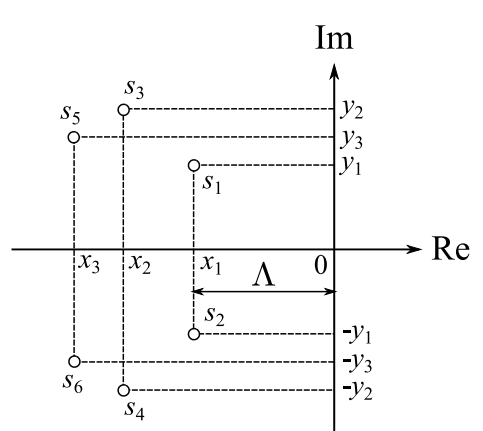

Fig. 2 Characteristic roots of a 3-DOF system. The real part of the characteristic roots (which is negative in a stable system) represents the speed of vibration decay, and the imaginary part represents the damped natural angular frequency. The stability $\Lambda$ describes how quickly the free vibration decays. 


\section{Previously published design expression: a problem and a question}

\subsection{A problem with the conventional method and one solution}

The number of parameters required in the design of a double-mass DVA based on the stability criterion is seven. This is because, in addition to the five design parameters $\left(\mu_{B}, v, v_{B}, \zeta_{2}, \zeta_{3}\right)$ of the DVA, the coordinate values $\left(x_{r}, y_{r}\right)$ are required if the characteristic equation takes a triple root. Once the value of $x_{r}$ is known, the maximized stability index is obtained from the expression $\Lambda_{\max }=-x_{r}$. Thus, the number of equations required by this method is seven, these equations being as follows (Asami and Yamada, 2020):

$$
\begin{aligned}
f_{1} & =3 x_{r}\left(1+\mu_{B}\right)+\zeta_{1}\left(1+\mu_{B}\right)+\zeta_{2}\left(1+\mu+\mu_{B}\right) v+\zeta_{3}\left[1+(1+\mu) \mu_{B}\right] v v_{B}=0 \\
f_{2} & =\left(1-15 x_{r}^{2}-3 y_{r}^{2}\right)\left(1+\mu_{B}\right)+v^{2}\left\{1+\mu+\mu_{B}+\left[1+(1+\mu) \mu_{B}\right] v_{B}^{2}\right\}+4 \zeta_{1} \zeta_{2}\left(1+\mu_{B}\right) v+4 \zeta_{1} \zeta_{3}\left(1+\mu_{B}\right) v v_{B} \\
& +4 \zeta_{2} \zeta_{3}(1+\mu)\left(1+\mu_{B}\right) v^{2} v_{B}=0 \\
f_{3} & =2 x_{r}\left(5 x_{r}^{2}+3 y_{r}^{2}\right)+\zeta_{1} v^{2}\left(1+v_{B}^{2}\right)+\zeta_{2} v\left[1+(1+\mu) v^{2} v_{B}^{2}\right]+\zeta_{3} v\left[1+(1+\mu) v^{2}\right] v_{B}+4 \zeta_{1} \zeta_{2} \zeta_{3} v^{2} v_{B}=0 \\
f_{4}= & -3\left(x_{r}^{2}+y_{r}^{2}\right)\left(5 x_{r}^{2}+y_{r}^{2}\right)+v^{2}\left[1+v_{B}^{2}+(1+\mu) v^{2} v_{B}^{2}\right]+4 \zeta_{1} \zeta_{2} v^{3} v_{B}^{2}+4 \zeta_{1} \zeta_{3} v^{3} v_{B}+4 \zeta_{2} \zeta_{3} v^{2} v_{B}=0 \\
f_{5}= & 3 x_{r}\left(x_{r}^{2}+y_{r}^{2}\right)^{2}+\zeta_{1} v^{4} v_{B}^{2}+\zeta_{2} v^{3} v_{B}^{2}+\zeta_{3} v^{3} v_{B}=0 \\
f_{6} & =-\left(x_{r}^{2}+y_{r}^{2}\right)^{3}+v^{4} v_{B}^{2}=0 \\
f_{7} & =30 x_{r}^{37}-297 x_{r}^{39}+123 x_{r}^{41}+528 x_{5}^{35} y_{r}^{2}+\ll 59582 \text { terms } \gg+192 y_{r}^{10} \zeta_{1}^{3} \mu^{2} v^{24}=0
\end{aligned}
$$

Of these equations, the first six are derived from the requirement that the characteristic equation (a sixth-order equation) of the 3-DOF system takes a triple root, and the last equation is derived from the requirement that a set of simultaneous equations consisting of the first six equations takes a repeated root with respect to the parameter $x_{r}$. In the method, the first six equations are not completely connected: a binary system of simultaneous equations remains at the end. Even so, the last equation is extremely long, which is a weak point in the numerical calculation.

Figure 3(a) shows an example of a solution obtained by numerically solving the simultaneous equations shown in Eq. (4). This figure shows how the ratio of the optimized damping ratios $\zeta_{2 \text { opt }}$ and $\zeta_{3 \text { opt }}$ of the two DVAs varies against the primary damping ratio $\zeta_{1}$. When the mass ratio of the DVA to the primary system, $\mu$, becomes as small as 0.02 , the curve oscillates violently, which means that the effect of numerical calculation error is manifested. The numerical error increases as $\zeta_{1}$ increases, and when $\zeta_{1}$ is greater than 0.4 , the error becomes clearly visible even in the curve for $\mu=0.05$. It can be seen from Fig. 3(a) that the optimal damping ratios of the two DVAs are very close to each other. Therefore, we decided to approximate these two values of the optimal damping ratio as equal. Figure 3(b) shows the deterioration rate of the stability of the vibratory system caused by the approximation. The terms $\Lambda_{\max , 5}$ and $\Lambda_{\max , 4}$ appearing in the vertical axis label of this figure indicate the maximum stability of the system when the number of parameters to be optimized is 5 and 4 , respectively. If we reduce the number of variables by equating $\zeta_{2 \text { opt }}$ and $\zeta_{3 \text { opt }}$, the value of the stability of the

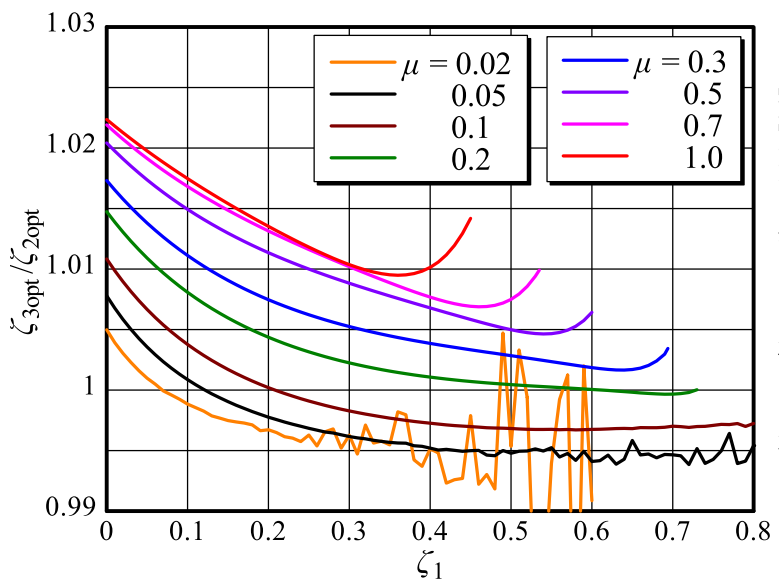

(a) Comparison of the magnitude of the damping ratios for two DVAs

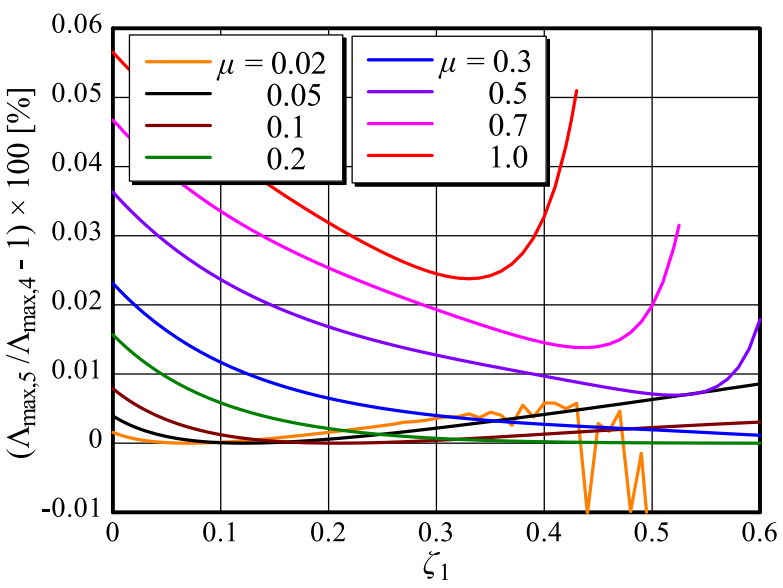

(b) Deterioration rate in stability when the number of parameters to be optimized is reduced from 5 to 4

Fig. 3 Optimal design conditions for a parallel double-mass DVA can only be obtained in the form of a numerical solution. The equation for calculating the numerical solution is very long, and depending on the conditions, the calculation error appears clearly in (a). In (a), the values of the optimal damping ratio of the two DVAs are very close (the ratio ranging from 0.995 to 1.02). Therefore, if the number of parameters to be optimized is reduced from 5 to 4 by setting these two damping ratios equal, this also reduces the value of the stability index, but the deterioration rate is less than $0.06 \%$, as shown in (b). 
system will always decrease. Therefore, the value of the ratio $\Lambda_{\max , 5} / \Lambda_{\max , 4}$ represented on the vertical axis will naturally be larger than 1. Figure 3(b) shows that the rate of deterioration in stability is less than $0.06 \%$ within the calculation range and that the deterioration rate is kept below $0.015 \%$ for $\mu$ less than 0.2 . Thus, reducing the number of unknown variables from seven to six has the advantage that the last long expression shown in Eq. (4) is no longer needed.

\subsection{A question about the optimized stability index}

Figure 4(a) shows the maximized evaluation index of three systems depicted in Fig. 1, optimized by the stability criterion. In this figure, there is no damping in the primary system $\left(\zeta_{1}=0\right)$, and the horizontal axis represents the mass ratio $\mu$ of the DVA to the primary system. As shown in this figure, the maximization index of the systems is improved in the order of those with single-mass, parallel-type double-mass, and series-type double-mass DVAs. If the DVA is designed based on the $H_{\infty}$ or $H_{2}$ criterion, then the vibration suppression performance of the parallel-type double-mass DVA is exactly halfway between those of the single-mass and the series-type double-mass DVAs (Asami, 2017). However, if the stability criterion is used for the DVA design, then the performance of the parallel-type double-mass DVA comes very close to that of the single-mass DVA.

Figure 4(b)-(d) show the frequency responses of three systems with a DVA of mass ratio $\mu=0.05$ optimized by the stability criterion. The optimal damping ratio of the DVA designed using the stability criterion becomes quite large, so the frequency response has only one resonant point. The height of this resonant point is determined by the value of stability, and it can be seen that the height of the resonant point of the system with a parallel-type double-mass DVA is only slightly reduced compared to that of the system with a single-mass DVA. Thus, another objective of this study is to find the reason why the stability of the system is only slightly improved by duplicating the DVA.

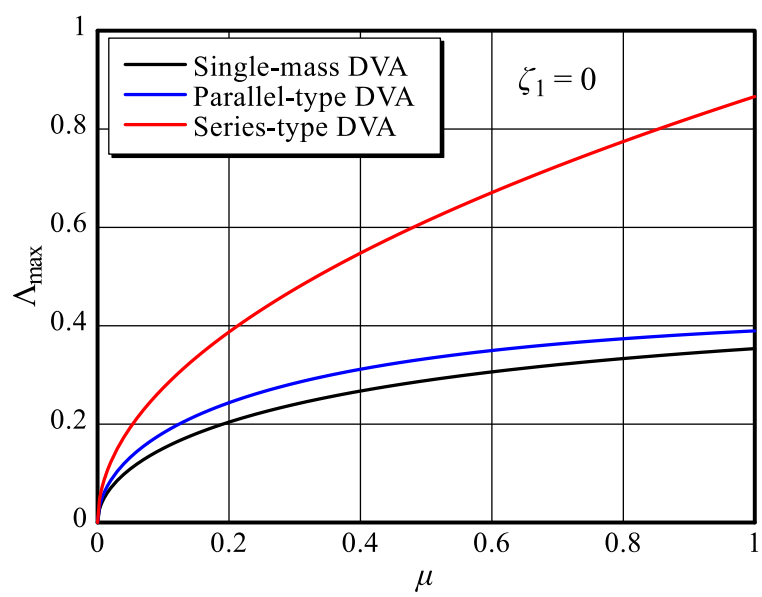

(a) Stability index $\Lambda_{\max }$ for three types of DVA

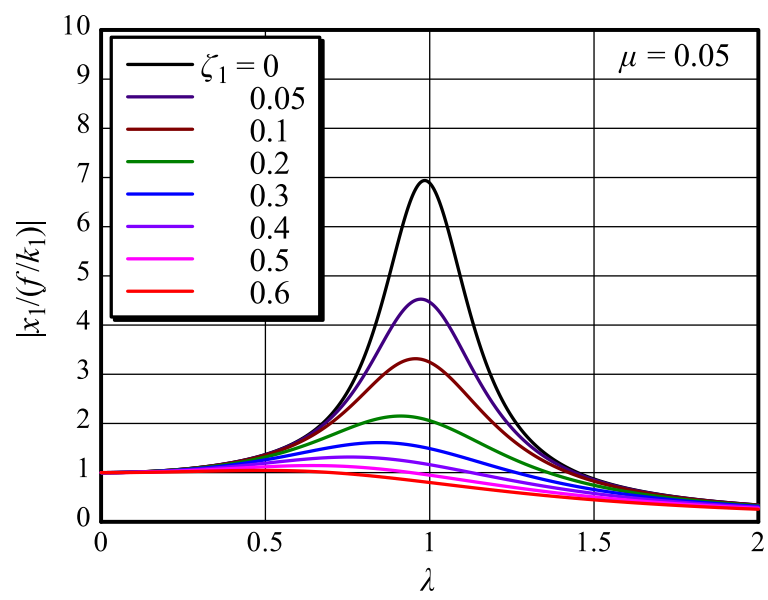

(c) Primary system with series-type double-mass DVA

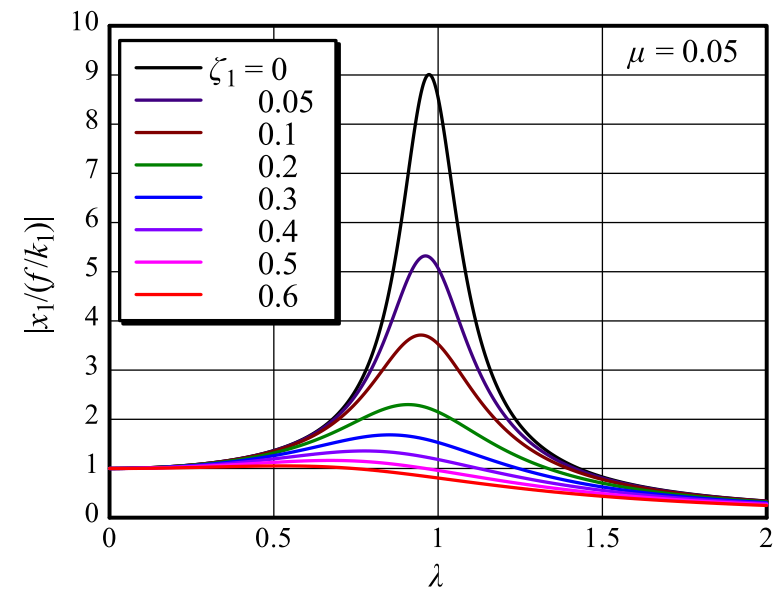

(b) Primary system with single-mass DVA

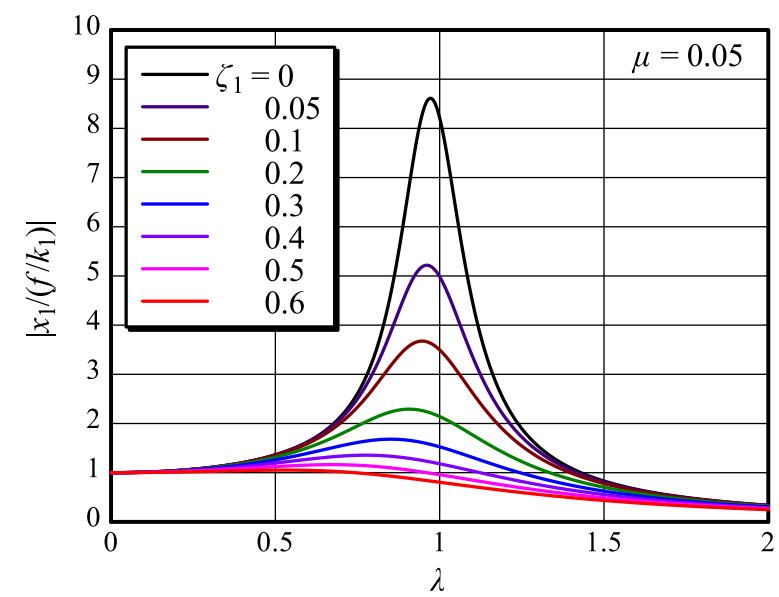

(d) Primary system with parallel-type double-mass DVA

Fig. 4 Improvement of vibration suppression performance by making the DVA double-mass. As shown in (a), the stability index is greatly improved for a series-type double-mass DVA, but only slightly improved for a parallel-type DVA. The magnitude of the stability index is reflected in the steady-state response of the system. The steady-state response of a system with a single-mass DVA with mass ratio $\mu=0.05$ is shown in (b). In the response (c) for the system with a series-type double-mass DVA with the same mass ratio, the resonant height is quite low, while the response (d) for the system with a parallel-type DVA is not so low. 


\section{Evaluation index of optimization and performance comparison of dynamic absorbers}

\subsection{Root trajectory of a single-degree-of-freedom system}

Before discussing the characteristic root trajectories of 2- and 3-DOF vibratory systems, we briefly review the root trajectory of the most basic SDOF system. Figure 5(a) shows the root trajectory for the SDOF system in Fig. 1(a) whose DVA is removed from the primary system $P$ in Fig. 1. In Fig. 5(a), the real and imaginary parts of the characteristic root are denoted by $x_{r}$ and $y_{r}$, respectively, and the linear distance from the coordinate origin is denoted by $r_{s}$. Characteristic roots usually have units of angular frequency, but the characteristic roots shown in this figure have been transformed to dimensionless parameters by dividing by the natural angular frequency $\omega_{1}$ of the primary system. In this way, the root trajectory for the underdamped system $\zeta_{1}<1$ is a semicircle with radius $r_{s}=1$. The stability index $\Lambda$ of the vibratory system is identical to the damping ratio $\zeta_{1}$ of the system. The characteristic roots appear in the second and third quadrants of the complex plane, but Fig. 5(a) shows only the characteristic roots that appear in the second quadrant. As is well known, when the damping ratio $\zeta_{1}$ of the vibratory system reaches zero, the characteristic root becomes a pure imaginary number (that is, the real part $x_{r}$ becomes zero). The characteristic root moves on a semicircle until the damping ratio of the system reaches the critical damping ratio $\zeta_{1}=1$, and it becomes a double root with $x_{r}=-1$ at the critical damping. At the critical damping, the stability index takes the maximum value $\Lambda_{\max }=1$; as the damping ratio further increases, the characteristic equation takes two different real roots, one of which moves toward the coordinate origin, and the stability of the system decreases accordingly. As will be clarified later, the stability of the system does not exceed 1 up to the 3-DOF system. Even if the degree of freedom of the system were further increased, this situation would not change.

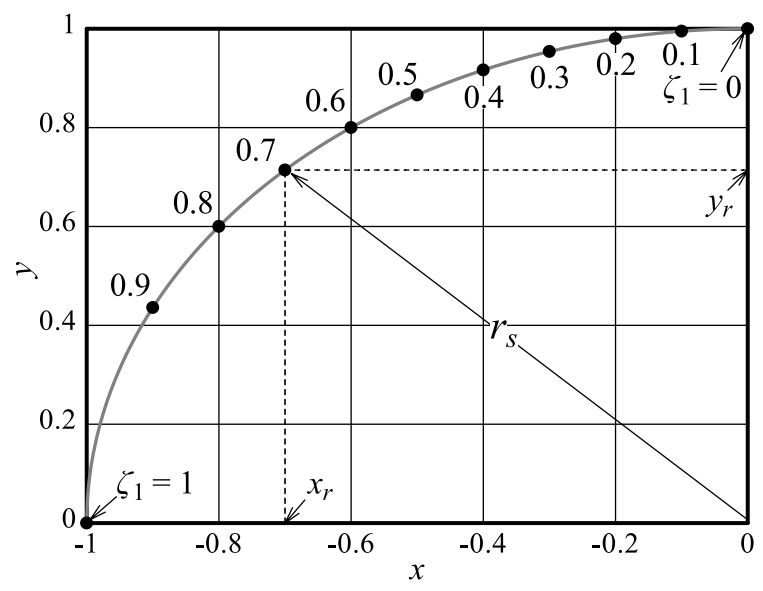

(a) SDOF system (System without DVA)

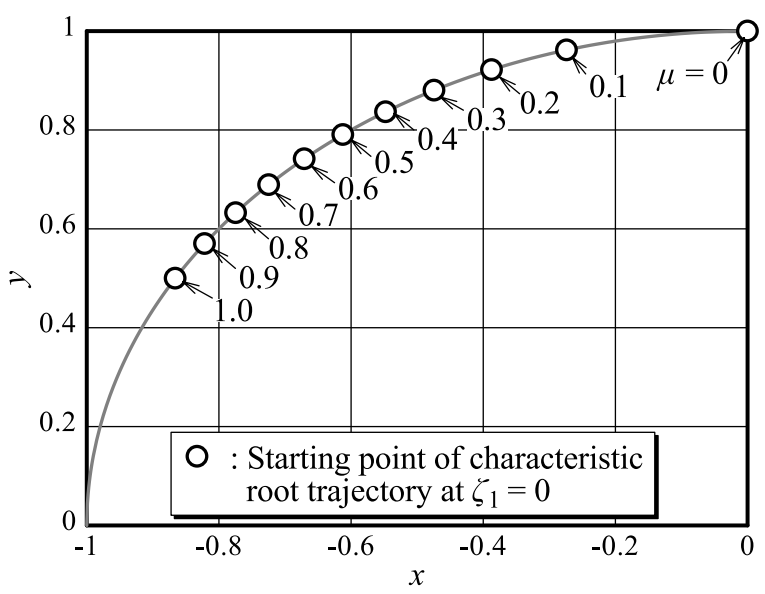

(c) System with series-type double-mass DVA

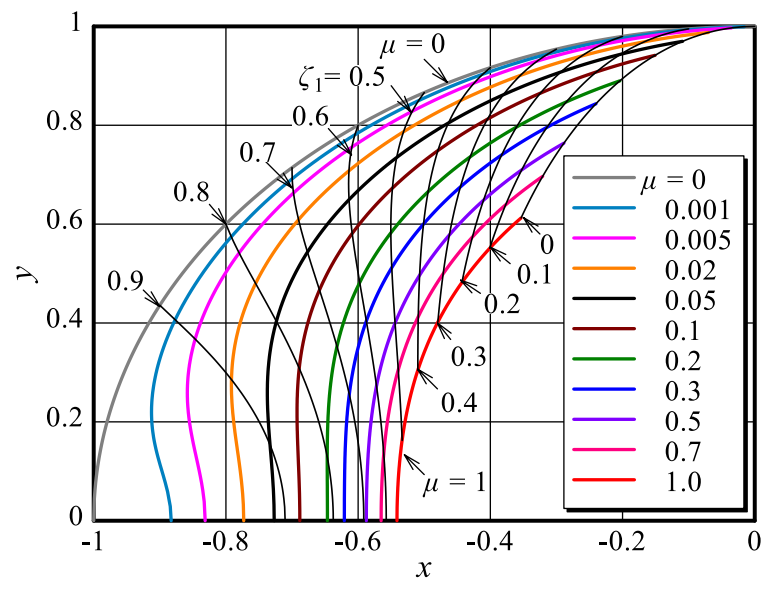

(b) System with single-mass DVA

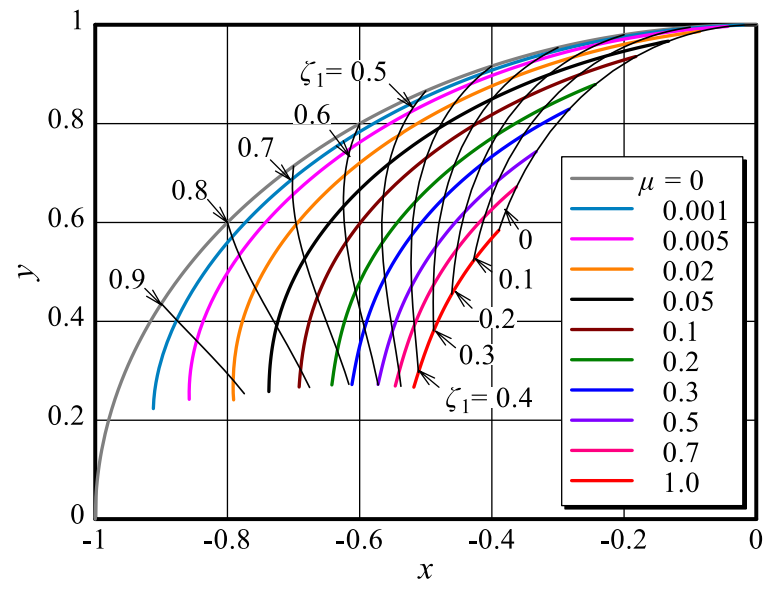

(d) System with parallel-type double-mass DVA

Fig. 5 Characteristic root trajectories that appear on the complex plane when the SDOF, 2-, and 3-DOF systems take a repeated root. In the SDOF system and the 3-DOF system with a series-type double-mass DVA, the root trajectory is an arc of radius $r_{s}=1$, as shown in (a) and (c), respectively. In contrast, for the 2-DOF system with a single-mass DVA and the 3-DOF system with a parallel-type double-mass DVA, the root trajectory is within the circle of radius $r_{s}=1$, as shown in (b) and (d), respectively. The major difference between (b) and (d) is that there is no trajectory in (d) having $y_{r}$ less than 0.25 . This is because the mass of one of the DVAs will be zero in this case. 


\subsection{Root trajectory of a system with a single-mass dynamic absorber}

Figure 5(b) shows the characteristic root trajectory that appears on the complex plane when the 2-DOF system shown in Fig. 1 (a) takes a double root. The gray curve labeled $\mu=0$ is the characteristic root trajectory of the SDOF system. When a DVA is attached to this SDOF system, the distance $r_{s}$ from the coordinate origin to the characteristic root becomes smaller than 1 (that is, moves inside the semicircle with radius 1 ). Then, until $\zeta_{1}$ reaches about 0.5 , the characteristic roots move to the left and the stability of the system increases; however, the stability decreases in the heavily damped system where $\zeta_{1}$ is greater than 0.5 . Furthermore, before reaching the critical damping ratio $\zeta_{1}=1$, the characteristic root reaches the real axis, momentarily becoming a quadruple root, and then splits into two different real double roots. The major difference from the SDOF system is that when the 2-DOF system takes two different real roots, both are to the right of the point -1 , and one reaches -0.5 and the other reaches zero at the critical damping ratio $\zeta_{1}=1$.

\subsection{Root trajectory of a system with a series-type double-mass dynamic absorber}

Figure 5(c) shows the root trajectory when the series-type 3-DOF system shown in Fig. 1(b) takes a triple root. The characteristic root moves on the semicircle of radius $r_{s}=1$ as in the case of the SDOF system. The difference from the SDOF system is that the real part of the trajectory starts from the zero position in the SDOF system, but it starts from a point to the left of the zero position (see white circles in Fig. 5(c)) in the 3-DOF system. As a result, the value of the primary damping ratio $\zeta_{1}$ when the characteristic root becomes a real number is smaller than 1 (see Fig. 8(a)).

\subsection{Root trajectory of a system with a parallel-type double-mass dynamic absorber}

Figure 5(d) shows the root trajectory when the parallel-type 3-DOF system shown in Fig. 1(c) takes a triple root. At first glance, it is obviously very similar to the root trajectory diagram shown in Fig. 5(b). In fact, the curves of $\mu=$ const. perfectly coincide with each other. The major difference between the two figures is that there is no root trajectory in Fig. 5(d) with $y_{r}$ less than a value that is about 0.25 . This is because the value of $\mu_{B \text { opt }}$ becomes zero at this point. From this point on, the parallel-type double-mass DVA becomes a single-mass DVA, and the curves of Fig. 5(d) continue as the corresponding $\mu$ curves in Fig. 5(b). Another difference between Fig. 5(d) and (b) is the curves of $\zeta_{1}=$ const., displayed in black. The starting points (upper ends) of the curves are the same in the two figures, but the curves in Fig. 5(d) then shift to the left. This means that the stability of the system with the parallel-type double-mass DVA is slightly higher than that with the single-mass DVA for the same mass ratio.

\subsection{Answer to the question in section 4.2}

We have arrived at the reason for the poor improvement of the stability index for a parallel-type double-mass DVA. When the characteristic roots are a repeated root, the linear distance $r_{s}$ of the root from the coordinate origin will never exceed 1. This maximum value can be reached in a system with a series-type double-mass DVA, but not in the case of a parallel-type DVA.

\section{Characteristic equation and coordinate calculation formula of characteristic roots}

\subsection{Characteristic equation}

The characteristic equation of the vibratory system shown in Fig. 1(c) is as follows:

$$
\begin{aligned}
D(s) & =a_{0} S^{6}+a_{1} S^{5}+a_{2} S^{4}+a_{3} S^{3}+a_{4} S^{2}+a_{5} S+a_{6}=0 \\
a_{0} & =1+\mu_{B}, \quad a_{1}=2\left\{\zeta_{1}\left(1+\mu_{B}\right)+v\left[\zeta_{2}\left(1+\mu+\mu_{B}\right)+\zeta_{3}\left(1+\mu_{B}+\mu \mu_{B}\right) v_{B}\right]\right\} \\
a_{2}= & 1+v^{2}+\mu v^{2}+4 \zeta_{2} \zeta_{3}(1+\mu) v^{2} v_{B}+v^{2} v_{B}^{2}+\mu_{B}+4 \zeta_{1} v\left(1+\mu_{B}\right)\left(\zeta_{2}+\zeta_{3} v_{B}\right) \\
& +\mu_{B} v^{2}\left[1+4 \zeta_{2} \zeta_{3}(1+\mu) v_{B}+(1+\mu) v_{B}^{2}\right] \\
a_{3}= & 2\left(1+\mu_{B}\right) v\left\{\zeta_{2}+\zeta_{1} v+v_{B}\left[\zeta_{3}+4 \zeta_{1} \zeta_{2} \zeta_{3} v+\zeta_{3} v^{2}(1+\mu)+\zeta_{1} v v_{B}+\zeta_{2}(1+\mu) v^{2} v_{B}\right]\right\} \\
a_{4}= & \left(1+\mu_{B}\right) v^{2}\left[1+4 \zeta_{3}\left(\zeta_{2}+\zeta_{1} v\right) v_{B}+v_{B}^{2}+4 \zeta_{1} \zeta_{2} v v_{B}^{2}+(1+\mu) v^{2} v_{B}^{2}\right] \\
a_{5}= & 2\left(1+\mu_{B}\right) v^{3} v_{B}\left(\zeta_{3}+\zeta_{2} v_{B}+\zeta_{1} v v_{B}\right), \quad a_{6}=\left(1+\mu_{B}\right) v^{4} v_{B}^{2},
\end{aligned}
$$

where the parameter $S\left(=s / \omega_{1}\right)$ shown here represents the dimensionless characteristic root. A previous study (Asami et al., 2018) showed that when the stability of a 3-DOF system is maximized, the characteristic equation takes a triple root. In this case, Eq. (5) should be factored as follows:

$$
D(s)=\left(1+\mu_{B}\right)\left[S-\left(x_{r}+i y_{r}\right)\right]^{3}\left[S-\left(x_{r}-i y_{r}\right)\right]^{3} .
$$

As discussed in Section 4.1, even if the damping ratios of the two DVAs are assumed to be equal, the error introduced by this approximation will be negligible. Furthermore, we will use the distance $r_{s}$ of the characteristic root from the 
coordinate origin instead of the coordinate $y_{r}$ of the characteristic root. In other words, the following substitutions are performed:

$$
\zeta_{3}=\zeta_{2}, \quad y_{r}=\sqrt{r_{s}^{2}-x_{r}^{2}}
$$

From a comparison of the coefficients in Eqs. (5) and (6), using Eq. (7), the following six identities are obtained:

$$
\begin{aligned}
f_{1} & =\left(3 x_{r}+\zeta_{1}\right)\left(1+\mu_{B}\right)+\zeta_{2} v\left[1+\mu+\mu_{B}+\left(1+\mu_{B}+\mu \mu_{B}\right) v_{B}\right]=0 \\
f_{2} & =\left(1+\mu_{B}\right)\left[1-3 r_{s}^{2}-12 x_{r}^{2}+4 \zeta_{1} \zeta_{2} v\left(1+v_{B}\right)\right] \\
& +(1+\mu) v^{2}\left[1+4 \zeta_{2}^{2}\left(1+\mu_{B}\right) v_{B}+\mu_{B} v_{B}^{2}\right]+v^{2}\left(\mu_{B}+v_{B}^{2}\right)=0 \\
f_{3} & =2 x_{r}\left(3 r_{s}^{2}+2 x_{r}^{2}\right)+v\left\{\zeta_{1} v\left(1+v_{B}^{2}\right)+4 \zeta_{1} \zeta_{2}^{2} v v_{B}+\zeta_{2}\left(1+v_{B}\right)\left[1+(1+\mu) v^{2} v_{B}\right]\right\}=0 \\
f_{4} & =-3 r_{s}^{2}\left(r_{s}^{2}+4 x_{r}^{2}\right)+v^{2}\left\{1+4 \zeta_{1} \zeta_{2} v v_{B}\left(1+v_{B}\right)+4 \zeta_{2}^{2} v_{B}+\left[1+(1+\mu) v^{2}\right] v_{B}^{2}\right\}=0 \\
f_{5} & =3 r_{s}^{4} x_{r}+v^{3} v_{B}\left[\zeta_{1} v v_{B}+\zeta_{2}\left(1+v_{B}\right)\right]=0 \\
f_{6} & =-\left(r_{s}^{3}-v^{2} v_{B}\right)\left(r_{s}^{3}+v^{2} v_{B}\right)=0
\end{aligned}
$$

In Eq. (8), two parameters $\left(\mu\right.$ and $\left.\zeta_{1}\right)$ are given and the remaining six $\left(\mu_{B}, v, v_{B}, \zeta_{2}, x_{r}\right.$, and $\left.r_{s}\right)$ are treated as unknown parameters. Because the number of independent equations is also six, the number of equations required for this optimization problem is sufficient.

\subsection{Solution for the undamped primary system}

If there is no damping in the primary system, the following solution can be obtained from Eq. (8)

$$
\begin{aligned}
& f_{r}=a_{1} r_{s}^{11}+a_{2} r_{s}^{10}+a_{3} r_{s}^{9}+a_{4} r_{s}^{8}+a_{5} r_{s}^{7}+a_{6} r_{s}^{6}+a_{7} r_{s}^{5}+a_{8} r_{s}^{4}+a_{9} r_{s}^{3}+a_{10} r_{s}^{2}+a_{11} r_{s}+a_{12}=0 \\
& a_{1}=64(1+\mu)^{3}, \quad a_{2}=-72(1+\mu)^{3}, \quad a_{3}=-(1+\mu)^{2}(301-19 \mu), \quad a_{4}=2(1+\mu)(221+225 \mu) \\
& a_{5}=12(1+\mu)(29-16 \mu), \quad a_{6}=-841-832 \mu+25 \mu^{2}, \quad a_{7}=16(11+31 \mu), \quad a_{8}=114(4-\mu) \\
& a_{9}=-377+9 \mu, \quad a_{10}=122, \quad a_{11}=-18, \quad a_{12}=1,
\end{aligned}
$$

and

$$
\left.\begin{array}{l}
v_{\mathrm{opt}}=\sqrt{\frac{r_{s}}{a}\left(b-\sqrt{b^{2}-r_{s}^{4} a^{2}}\right)}, \quad \zeta_{2 \mathrm{opt}}=\frac{\left(3 r_{s}\right)^{3 / 2} v_{\mathrm{opt}}}{2\left(r_{s}^{3}+v_{\mathrm{opt}}^{2}\right)} \sqrt{1-2 r_{s}+r_{s}^{3}(1+\mu)} \\
\mu_{B \mathrm{opt}}=\frac{r_{s}^{4}-r_{s}^{3}-\left(1-r_{s}-r_{s} \mu\right) v_{\mathrm{opt}}^{2}}{r_{s}^{3}\left(1-r_{s}-r_{s} \mu\right)+\left(1-r_{s}\right) v_{\mathrm{opt}}^{2}}, \quad v_{B \mathrm{opt}}=\frac{r_{s}^{3}}{v_{\mathrm{opt}}^{2}}, \quad x_{r}=-\frac{\zeta_{2 \mathrm{opt}}\left(r_{s}^{3}+v_{\mathrm{opt}}^{2}\right)}{3 r_{s} v_{\mathrm{opt}}}, \quad \Lambda_{\max }=-x_{r} \\
\text { where } a=-2\left(1-r_{s}-r_{s} \mu\right), \quad b=1-9 r_{s}+17 r_{s}^{2}+r_{s}^{3}(7+8 \mu)-24 r_{s}^{4}(1+\mu)+8 r_{s}^{6}(1+\mu)^{2} .
\end{array}\right\}
$$

Unfortunately, one parameter, $r_{s}$, cannot be solved algebraically and requires solving the eleventh-order algebraic equation given in Eq. (9). Equation (9) yields 11 different values of $r_{s}$, but there is only one physically meaningful value, namely the largest real value not exceeding 1.

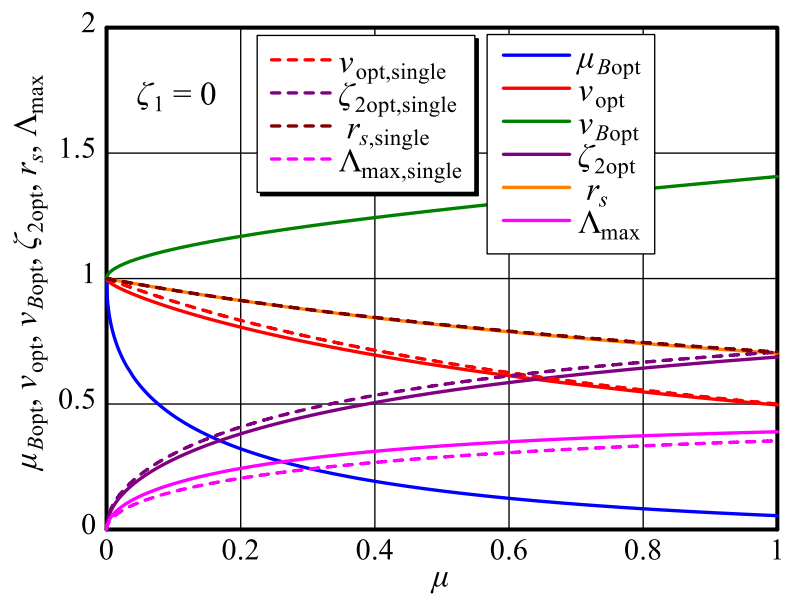

Fig. 6 Optimal design conditions for a parallel-type double-mass DVA and a single-mass DVA mounted on undamped primary systems. It is noteworthy that the values of $r_{s}$ for the two DVAs are in the immediate vicinity of each other. For the parallel-type DVA, the value of $r_{s}$ is obtained as one of the solutions to an eleventh-order algebraic equation, but if it is approximated by $r_{s}=\sqrt{1 /(1+\mu)}$ for the single-mass DVA, the numerical error is amplified and $\mu_{B \text { opt }}$ becomes zero for all values of $\mu$. To take this into account, the values of $\mu_{B \text { opt }}$ in the figure were calculated to an accuracy of 9 digits. 
Figure 6 shows the value of $r_{s}$ obtained by solving Eq. (9), the optimal design condition for a parallel-type DVA obtained by substituting this value into Eq. (10), and the maximized stability index $\Lambda_{\max }$ plotted against the mass ratio $\mu$. Many real roots of $r_{s}$ can be obtained from Eq. (9). However, only the value of $r_{s}$ shown in Fig. 6 makes all four design conditions in the DVA real positive numbers. The dashed lines shown in Fig. 6 are the optimal design conditions and values of $r_{s}$ and $\Lambda_{\max }$ for a single-mass DVA. These values are calculated from the following:

$$
v_{\mathrm{opt}, \text { single }}=\frac{1}{1+\mu}, \quad \zeta_{\text {2opt,single }}=\sqrt{\frac{\mu}{1+\mu}}, \quad r_{s, \text { single }}=\sqrt{\frac{1}{1+\mu}}, \quad \Lambda_{\text {max }, \text { single }}=\frac{1}{2} \sqrt{\frac{\mu}{1+\mu}} .
$$

Comparing the dashed and corresponding solid lines in Fig. 6, we can see that they are very close to each other. In particular, for the linear distance $r_{s}$ from the coordinate origin to the characteristic root, the solid and dashed lines appear to overlap. In fact, for $\mu=0.1, r_{s, \text { single }}=0.953463$ compared to $r_{s}=0.953327$, and for $\mu=0.5, r_{s, \text { single }}=0.816497$ compared to $r_{s}=0.814410$, indicating the same value up to the second or third digit. However, if we approximate the formula for $r_{s}$ by $\sqrt{1 /(1+\mu)}$ just because these values are close together, the error is amplified and we end up with $\mu_{B \text { opt }}=0$ for all ranges of $\mu$. This means that this small difference is actually significant.

\subsection{Solution for the damped primary system}

If there is damping in the primary system, the following set of simultaneous equations can be obtained from Eq. (8):

$$
\begin{aligned}
f_{r}= & b_{1} r_{s}^{11}+b_{2} r_{s}^{10}+b_{3} r_{s}^{9}+b_{4} r_{s}^{8}+b_{5} r_{s}^{7}+b_{6} r_{s}^{6}+b_{7} r_{s}^{5}+b_{8} r_{s}^{4}+b_{9} r_{s}^{3}+b_{10} r_{s}^{2}+b_{11} r_{s}+b_{12}=0 \\
f_{x}= & 4 x_{r}^{3}+12 r_{s}^{2} \zeta_{1} x_{r}^{2}-3 r_{s}\left[1-2 r_{s}+r_{s}^{3}\left(1-4 \zeta_{1}^{2}+\mu\right)\right] x_{r}-r_{s}^{3} \zeta_{1}\left[1-3 r_{s}+2 r_{s}^{3}\left(1-2 \zeta_{1}^{2}+\mu\right)\right]=0 \\
b_{1} & =(1+\mu)\left\{3 x_{r}\left(1+\mu-4 \zeta_{1}^{2}\right)^{2}+\zeta_{1}\left[3+5 \mu+2 \mu^{2}-4 \zeta_{1}^{2}(2+\mu)\right]\right\} \\
b_{2} & =3 \zeta_{1}(1+\mu)\left(1+\mu-4 \zeta_{1}^{2}\right) \\
b_{3}= & -3(1+\mu)\left\{\zeta_{1}\left(4+3 \mu-4 \zeta_{1}^{2}\right)+24 x_{r}^{2} \zeta_{1}\left(1+\mu-4 \zeta_{1}^{2}\right)+x_{r}\left[4+3 \mu-\mu^{2}-4 \zeta_{1}^{2}(5+\mu)+16 \zeta_{1}^{4}\right]\right\} \\
b_{4}= & 12 x_{r}^{2} \zeta_{1}(1+\mu)\left(1+\mu-4 \zeta_{1}^{2}\right)-\zeta_{1}\left[12+18 \mu+5 \mu^{2}-4 \zeta_{1}^{2}(4+3 \mu)\right]+3 x_{r}\left[1+\mu-4 \zeta_{1}^{2}(5+4 \mu)-16 \zeta_{1}^{4}\right] \\
b_{5}= & 24 x_{r}^{2} \zeta_{1}(1+\mu)\left(10+3 \mu-10 \zeta_{1}^{2}\right)-72 x_{r}^{3}(1+\mu)\left(1+\mu-10 \zeta_{1}^{2}\right)+6 \zeta_{1}\left(3+2 \mu+2 \zeta_{1}^{2}\right)+9 x_{r}\left(2+\mu-\mu^{2}-4 \zeta_{1}^{2}\right) \\
b_{6}= & 6\left\{\zeta_{1}\left(3+2 \mu-2 \zeta_{1}^{2}\right)+6 x_{r}^{3}(1+\mu)\left(1+\mu-8 \zeta_{1}^{2}\right)-6 x_{r}^{2} \zeta_{1}\left(6+5 \mu+8 \zeta_{1}^{2}\right)-x_{r}\left[2+2 \mu+\mu^{2}-4 \zeta_{1}^{2}(1+\mu)-8 \zeta_{1}^{4}\right]\right\} \\
b_{7}= & -24 x_{r}^{2} \zeta_{1}\left(3+\mu-2 \zeta_{1}^{2}\right)+864 x_{r}^{4} \zeta_{1}(1+\mu)-\zeta_{1}\left(12-\mu+8 \zeta_{1}^{2}\right)-3 x_{r}\left(4-\mu-40 \zeta_{1}^{2}\right) \\
& +72 x_{r}^{3}\left[4+5 \mu+\mu^{2}-2 \zeta_{1}^{2}(4+3 \mu)\right] \\
b_{8}= & 3\left[3 x_{r}\left(2+\mu-4 \zeta_{1}^{2}\right)-4 \zeta_{1}-192 x_{r}^{4} \zeta_{1}(1+\mu)+4 x_{r}^{2} \zeta_{1}\left(3+\mu+20 \zeta_{1}^{2}\right)-12 x_{r}^{3}\left(10+9 \mu+16 \zeta_{1}^{2}\right)\right] \\
b_{9} & =3\left[x_{r}+\zeta_{1}+60 x_{r}^{2} \zeta_{1}-16 x_{r} \zeta_{1}^{2}-12 x_{r}^{3}\left(1-12 \zeta_{1}^{2}\right)+144 x_{r}^{5}(1+\mu)-48 x_{r}^{4} \zeta_{1}(5+2 \mu)\right] \\
b_{10} & =-3\left[4 x_{r}-\zeta_{1}+16 x_{r}^{2} \zeta_{1}+96 x_{r}^{4} \zeta_{1}+144 x_{r}^{5}(1+\mu)-12 x_{r}^{3}\left(5+\mu+8 \zeta_{1}^{2}\right)\right] \\
b_{11} & =12 x_{r}^{2}\left(1-12 x_{r}^{2}\right)\left(3 x_{r}-5 \zeta_{1}\right), \quad b_{12}=3 x_{r}\left(1-12 x_{r}^{2}\right)^{2} .
\end{aligned}
$$

By solving this set of simultaneous equations, the optimal design condition for the DVA can be calculated using

$$
\left.\begin{array}{l}
v_{\mathrm{opt}}=r_{s}^{3 / 2} \sqrt{-\frac{d_{2}+\sqrt{d_{2}^{2}-d_{1}^{2}}}{d_{1}}}, \quad \mu_{B \mathrm{opt}}=\frac{\mu_{B \mathrm{Num}}}{\mu_{B \mathrm{Den}}}, \quad v_{B \mathrm{opt}}=\frac{r_{s}^{3}}{v_{\mathrm{opt}}^{2}} \\
\zeta_{2 \mathrm{opt}}=\zeta_{3 \mathrm{opt}}=-\frac{\left(3 x_{r}+\zeta_{1}\right)\left(1+\mu_{B \mathrm{opt}}\right)}{v_{\mathrm{opt}}\left[1+\mu+\mu_{B \mathrm{opt}}+\left(1+\mu_{B \mathrm{opt}}+\mu \mu_{B \mathrm{opt}}\right) v_{B \mathrm{opt}}\right]},
\end{array}\right\}
$$

where

$$
\begin{aligned}
& d_{1}= 3 x_{r}-3 r_{s}^{2} x_{r}+3 r_{s}^{3} x_{r}-3 r_{s}^{5} x_{r}-36 x_{r}^{3}+36 r_{s} x_{r}^{3}+3 r_{s}^{2} \zeta_{1}+3 r_{s}^{3} \zeta_{1}-3 r_{s}^{4} \zeta_{1}-3 r_{s}^{5} \zeta_{1}-24 r_{s} x_{r}^{2} \zeta_{1}-12 r_{s}^{2} x_{r}^{2} \zeta_{1} \\
&+36 r_{s}^{3} x_{r}^{2} \zeta_{1}-12 r_{s}^{3} x_{r} \zeta_{1}^{2}+12 r_{s}^{5} x_{r} \zeta_{1}^{2}-3 r_{s}^{3} x_{r} \mu-3 r_{s}^{5} x_{r} \mu-2 r_{s}^{5} \zeta_{1} \mu \\
& d_{2}= 3 x_{r}-3 r_{s}^{2} x_{r}+3 r_{s}^{3} x_{r}-3 r_{s}^{5} x_{r}-36 x_{r}^{3}+18 r_{s} x_{r}^{3}+54 r_{s}^{2} x_{r}^{3}+3 r_{s}^{2} \zeta_{1}+3 r_{s}^{3} \zeta_{1}-3 r_{s}^{4} \zeta_{1}-3 r_{s}^{5} \zeta_{1}-42 r_{s} x_{r}^{2} \zeta_{1} \\
&-12 r_{s}^{2} x_{r}^{2} \zeta_{1}+54 r_{s}^{4} x_{r}^{2} \zeta_{1}-24 r_{s}^{3} x_{r} \zeta_{1}^{2}+6 r_{s}^{5} x_{r} \zeta_{1}^{2}+18 r_{s}^{6} x_{r} \zeta_{1}^{2}-2 r_{s}^{5} \zeta_{1}^{3}+2 r_{s}^{8} \zeta_{1}^{3}-3 r_{s}^{3} x_{r} \mu-3 r_{s}^{5} x_{r} \mu \\
&+54 r_{s}^{2} x_{r}^{3} \mu-2 r_{s}^{5} \zeta_{1} \mu+54 r_{s}^{4} x_{r}^{2} \zeta_{1} \mu+18 r_{s}^{6} x_{r} \zeta_{1}^{2} \mu+2 r_{s}^{8} \zeta_{1}^{3} \mu \\
& \mu_{B \text { Num }}=3 r_{s}^{4} x_{r}+r_{s}^{6} \zeta_{1}-\left(3 x_{r}+\zeta_{1}\right) v_{\mathrm{opt}}^{2}+3 r_{s} x_{r}(1+\mu) v_{\mathrm{opt}}^{2}-r_{s}^{3}\left\{3 x_{r}+\zeta_{1}\left[1-(1+\mu) v_{\mathrm{opt}}^{2}\right]\right\} \\
& \mu_{B \text { Den }}=3 r_{s}^{4} x_{r}(1+\mu)+r_{s}^{6} \zeta_{1}(1+\mu)+3 r_{s} x_{r} v_{\mathrm{opt}}^{2}-\left(3 x_{r}+\zeta_{1}\right) v_{\mathrm{opt}}^{2}-r_{s}^{3}\left[3 x_{r}+\zeta_{1}\left(1-v_{\mathrm{opt}}^{2}\right)\right]
\end{aligned}
$$


Figure 7 shows the optimal design condition for a parallel-type double-mass DVA attached to a damped primary system. As shown in Fig. 7(a), the optimal mass ratio $\mu_{B \text { opt }}$ of the two DVAs decreases steeply with an increase in the primary damping, and it becomes completely zero at a certain value of $\zeta_{1}$. Therefore, there is no solution for primary damping larger than this value. The points at which the curves in Figs. 7(b) to (d) terminate are where $\mu_{B \text { opt }}$ becomes zero.

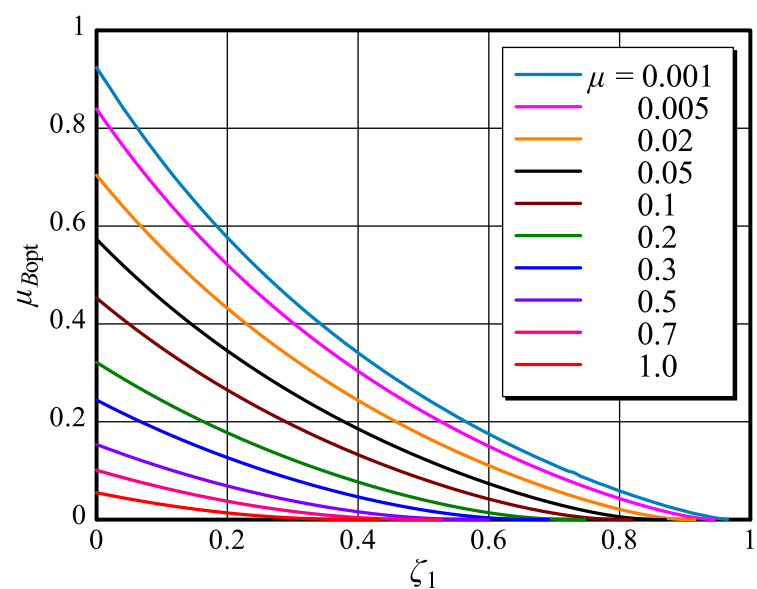

(a) Optimal mass ratio $\mu_{B \mathrm{opt}}$

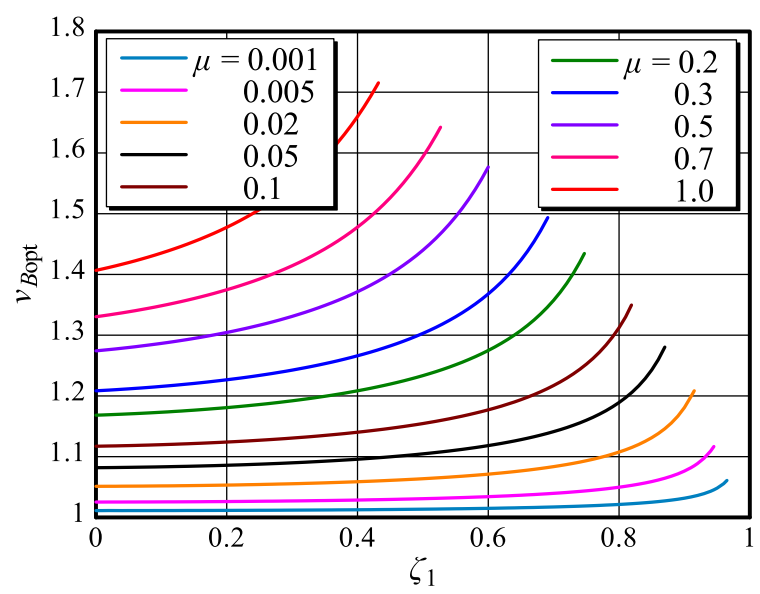

(c) Optimal tuning ratio $v_{B o p t}$

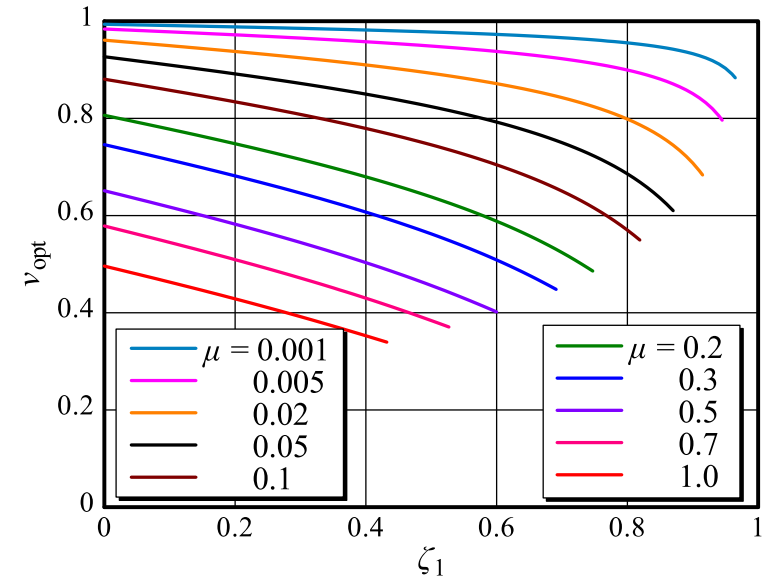

(b) Optimal tuning ratio $v_{\mathrm{opt}}$

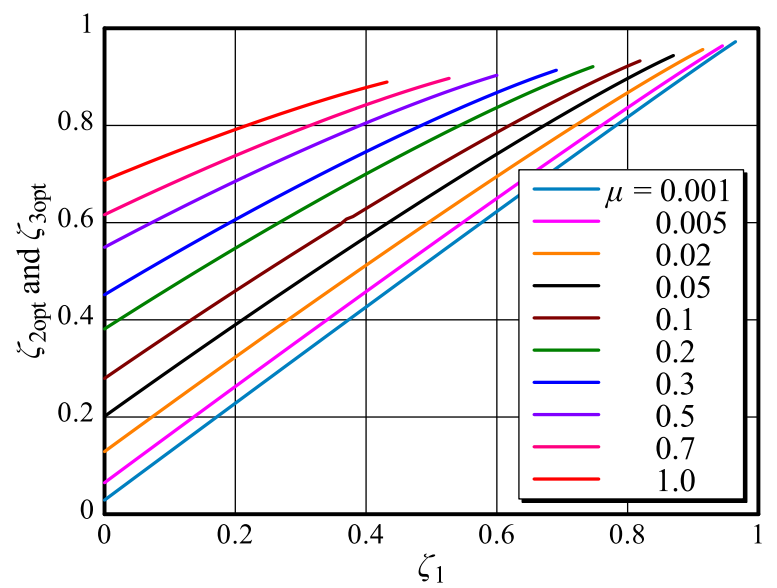

(d) Optimal damping ratio $\zeta_{2 \mathrm{opt}}=\zeta_{3 \mathrm{opt}}$

Fig. 7 Optimal parameters for the parallel-type double-mass DVA based on the stability criterion. To reduce the number of parameters to be optimized from five to four, the optimal damping ratios of the two DVAs are assumed to be approximately equal. The optimal mass ratio $\mu_{B o p t}$ of the two DVAs is exactly zero at a certain primary damping ratio $\zeta_{1}$, and thereafter it is a single-mass DVA.

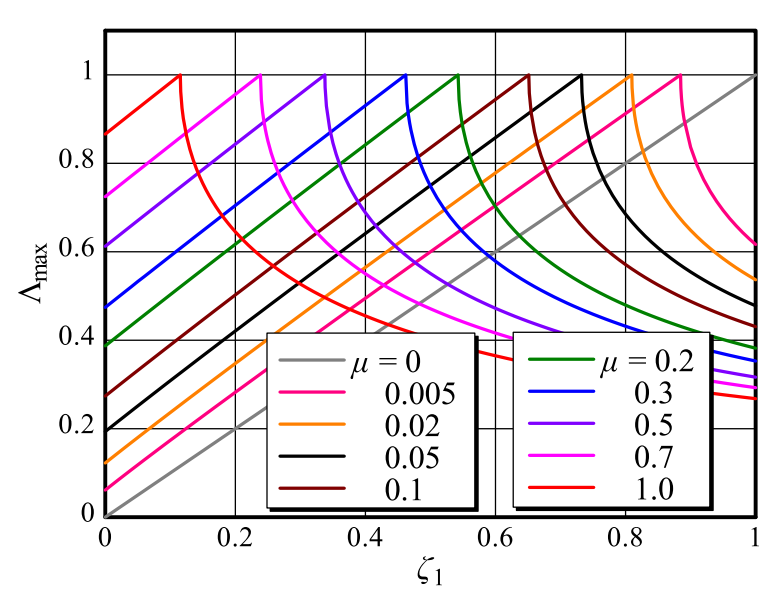

(a) Maximized stability for the series-type DVA

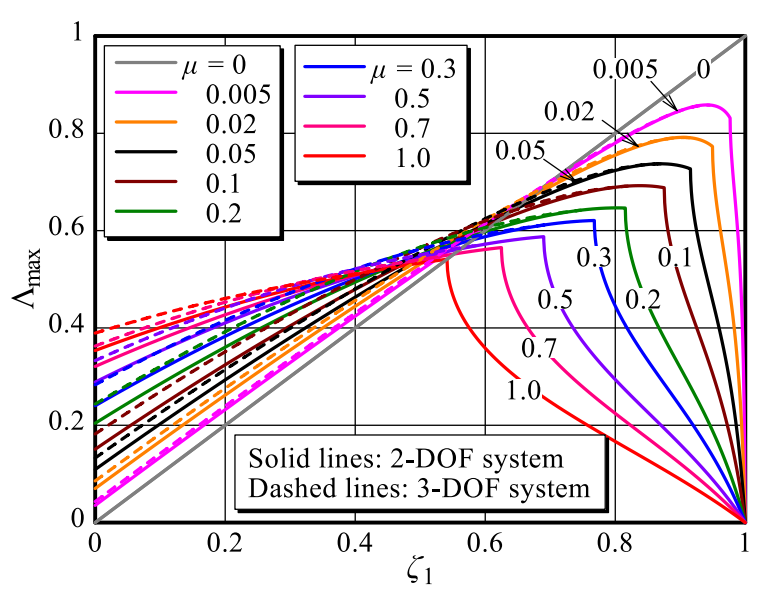

(b) Maximized stability for the parallel-type DVA

Fig. 8 Maximized stabilities of vibratory systems fitted with double-mass DVAs. The stability of the system fitted with the series-type double-mass DVA shown in (a) takes a maximum value when the characteristic roots reach the real axis, and thereafter, the roots move toward the coordinate origin. The stability of the system with the parallel-type double-mass DVA is indicated by the dashed lines in (b). As $\zeta_{1}$ increases, this stability approaches the stability of the single-mass DVA system (solid lines) and eventually merges. 
Figure 8(a) shows the maximized stability index of a system fitted with a series-type double-mass DVA. When two DVAs are attached in series to a damped primary system, the trajectories of dimensionless characteristic roots draw a semicircle with a radius of 1 , as shown in Fig. 5(c). Therefore, its stability index always reaches the maximum value of 1 , then suddenly decreases with increasing damping ratio $\zeta_{1}$, and finally converges to zero when the primary system damping ratio becomes infinity. The stability trajectory moves to the left as mass ratio $\mu$ increases, and falls suddenly at a smaller value of $\zeta_{1}$.

The maximized stability index $\Lambda_{\max }$ of the system with a parallel-type double-mass DVA is indicated by the dashed lines in Fig. 8(b). $\Lambda_{\max }$ is equal to $-x_{r}$, and the value of $x_{r}$ can be found as one solution to Eq. (12). As $\zeta_{1}$ increases, this stability approaches the stability of the single-mass DVA system (solid lines) and eventually merges. Comparing Fig. 8(a) and (b), it can be clearly understood that the stability of the system with the parallel-type double-mass DVA is much worse than that of the system with the series-type DVA.

\section{Responses of a system fitted with an optimized dynamic vibration absorber}

\subsection{Free-vibration response}

Figure 9 shows the free-vibration response of a system with a parallel-type double-mass DVA with $\mu=0.1$ after the primary mass starts moving at an initial velocity $v_{0}$. The Fourier series expansion method, developed by one of the authors (Yamada, 2020), is used to calculate the free vibration. Figure 9(c) and (d) show the response of a system with the DVA optimally designed by the stability criterion. As can be seen from the values in the figure legends, (c) is the free-vibration response of a system with a DVA designed strictly to account for the difference in the values of $\zeta_{2}$ and $\zeta_{3}$, and (d) is the response of the system with an approximately designed DVA obtained assuming that these two values are equal. Comparing (c) and (d), we can see that there is almost no difference between them. This indicates that the accuracy of the approximate design formulas shown in Eq. (10) is extremely high.

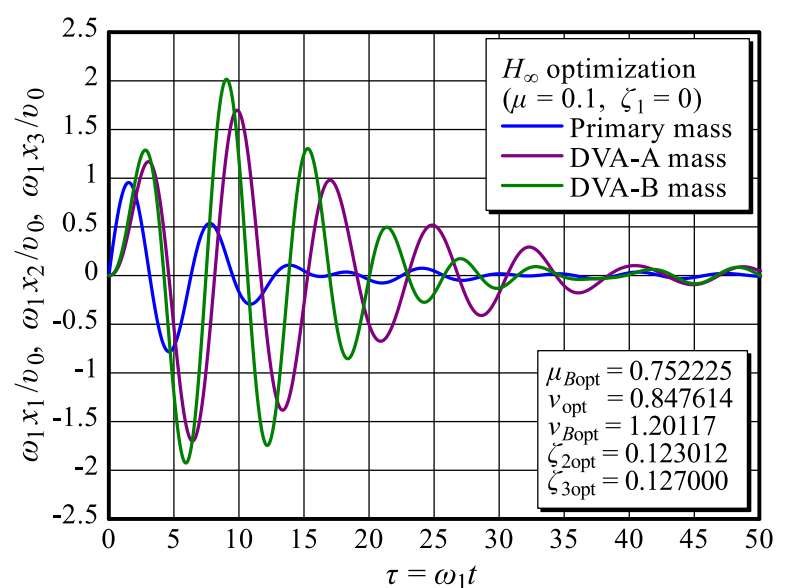

(a) $H_{\infty}$ optimization of a 3 -DOF system $(\mu=0.1)$

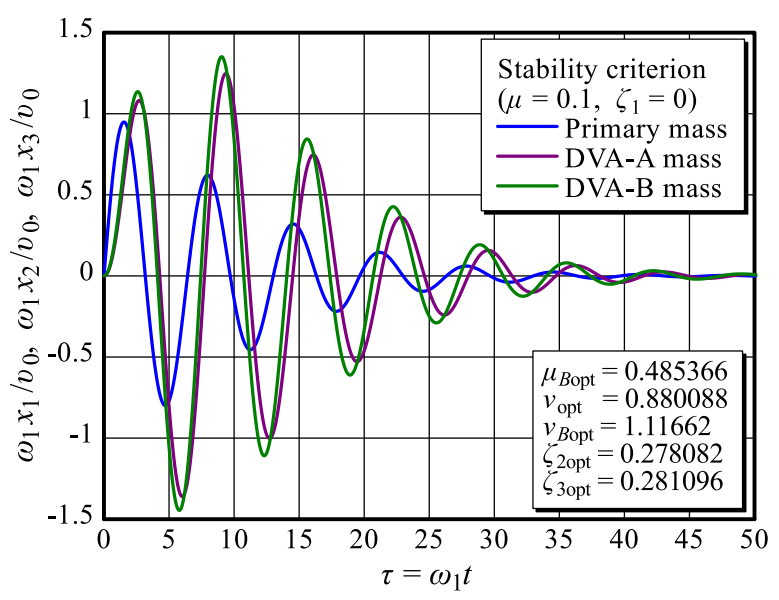

(c) Stability design of a 3-DOF system $(\mu=0.1)$

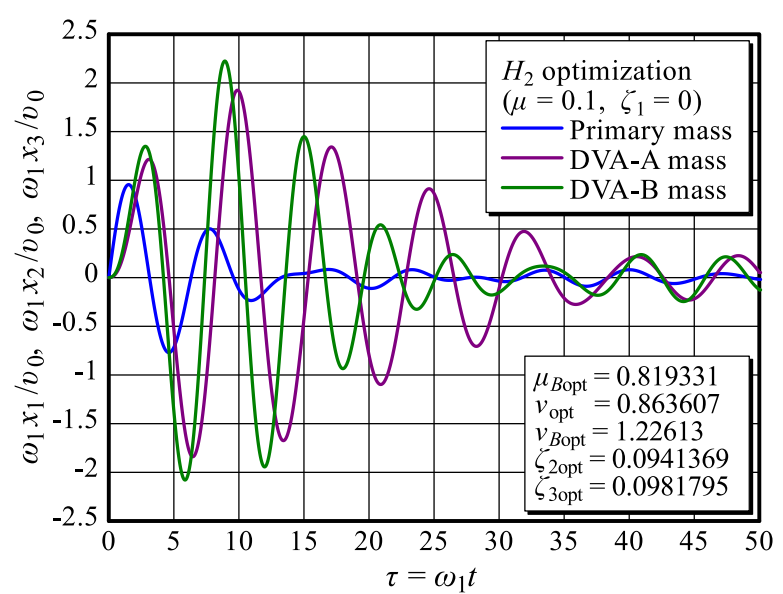

(b) $H_{2}$ optimization of a 3-DOF system $(\mu=0.1)$

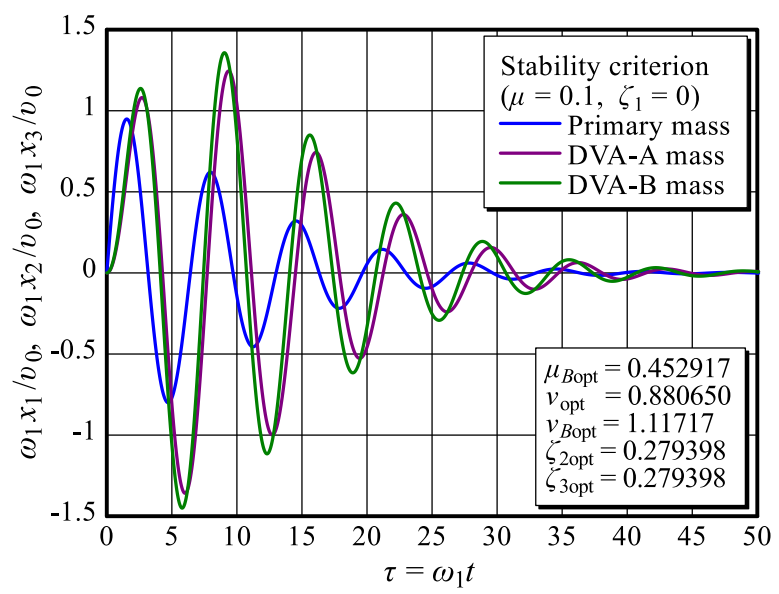

(d) Stability design of a 3-DOF system $(\mu=0.1)$

Fig. 9 Free-vibration responses of a system with the parallel-type double-mass DVA designed by three different optimization criteria. The initial damping of the primary mass is faster in systems with the DVA optimized by the $H_{\infty}$ or $H_{2}$ criterion, but the final termination of the vibration is delayed due to energy backflow from the DVA to the primary system. (d) is the response of a system with a simple design of the DVA with a reduced number of parameters, which is the same as the response (c) of a precisely designed system. 
Figure 9(a) and (b) show the free-vibration response of the system with a double-mass DVA designed with the other two optimization criteria, namely $H_{\infty}$ and $H_{2}$ optimizations. Comparing (a) and (b) with (c) or (d), the following interesting phenomenon can be observed: the initial attenuation of vibration is faster in (a) and (b), but the final termination of the vibration is faster in (c) or (d). This is because in the system with the DVA designed by the stability criterion, the vibration amplitudes of the primary mass and the absorber mass decrease at the same rate, and there is no energy backflow from the DVA to the primary system, or vice versa. Another way to look at this is that in a system optimally designed with the stability criterion, the phase relationship between the primary system and the two DVAs is fixed, and the primary mass always vibrates before the DVA masses.

\subsection{Steady-state response}

Figure 10 shows the steady-state response of a system fitted with a parallel-type double-mass DVA optimally designed with the three criteria. The four panels of Fig. 10 are arranged in the same way as those in Fig. 9, showing the system with the DVA designed by (a) the $H_{\infty}$, (b) the $H_{2}$, and (c) and (d) the stability criteria. Again, the response (c) of the system with the DVA designed strictly using the stability criterion is almost the same as the response (d) of the system with the DVA designed approximately using Eq. (13). A characteristic feature of the steady-state response of the system with the DVA optimized by the stability criterion is that only one resonance point appears in the multi-degree-of-freedom system. This is due to the fact that the damping ratios of the DVA are particularly large compared those of the other criteria, as indicated in the legends - in the upper left corners of the graphs.

The system designed by the stability criterion has the disadvantage of sharp resonance as shown in Fig. 10, but it also has the advantage of suppressing the relative displacement between the primary system and the DVAs as shown in Fig. 9 , which reduces the fatigue failure of the springs.

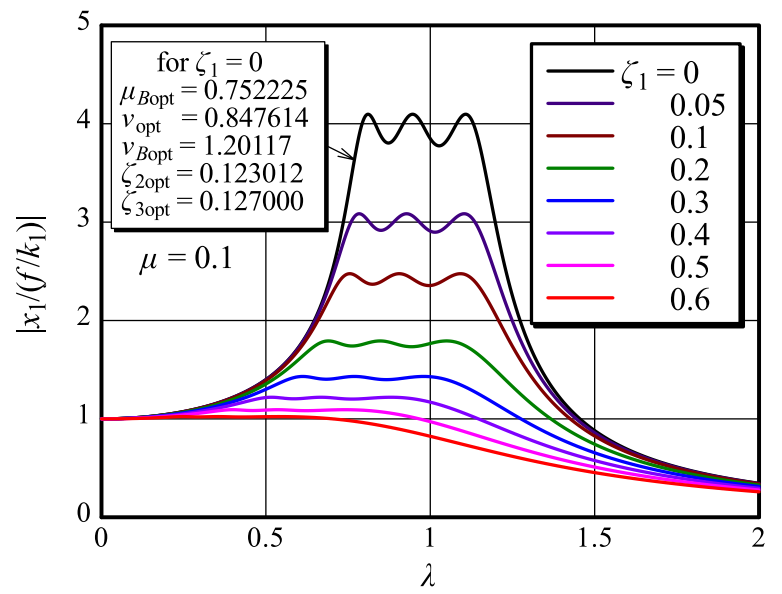

(a) $H_{\infty}$ optimization of a parallel-type 3-DOF system $(\mu=0.1)$

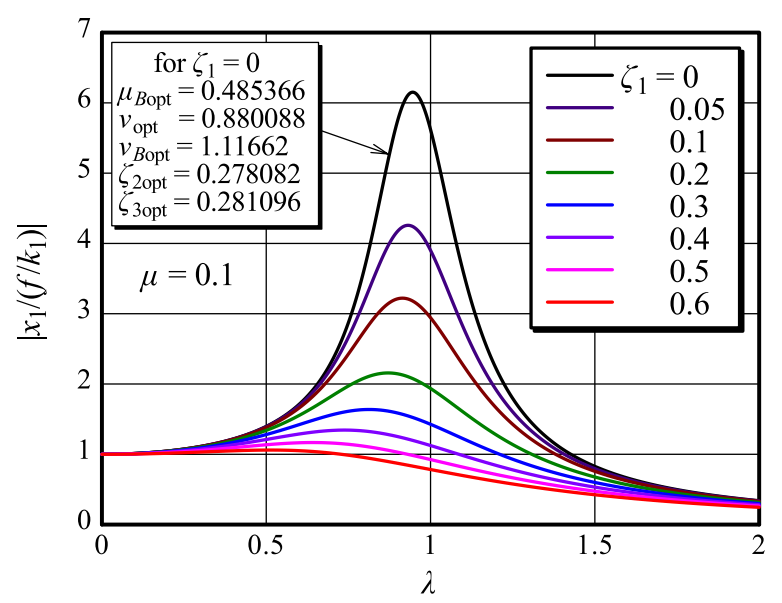

(c) Stability criterion of a parallel-type 3-DOF system $(\mu=0.1)$

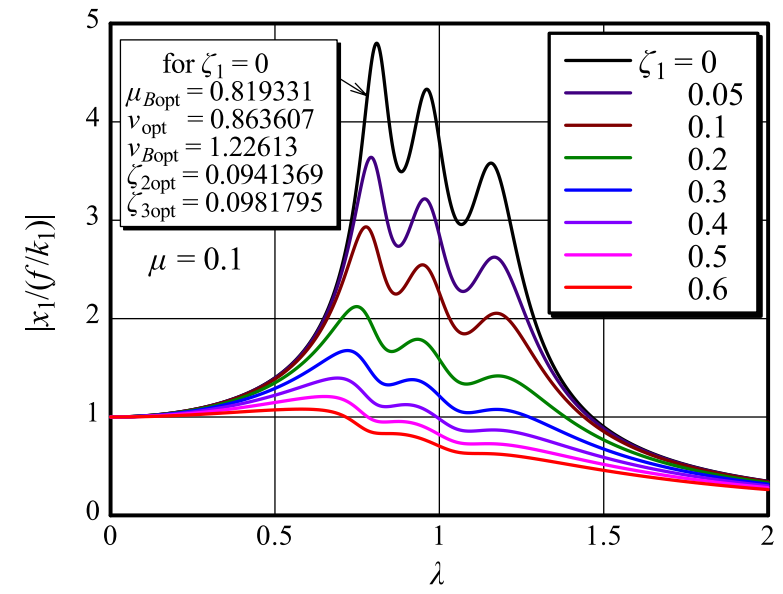

(b) $H_{2}$ optimization of a parallel-type 3-DOF system $(\mu=0.1)$

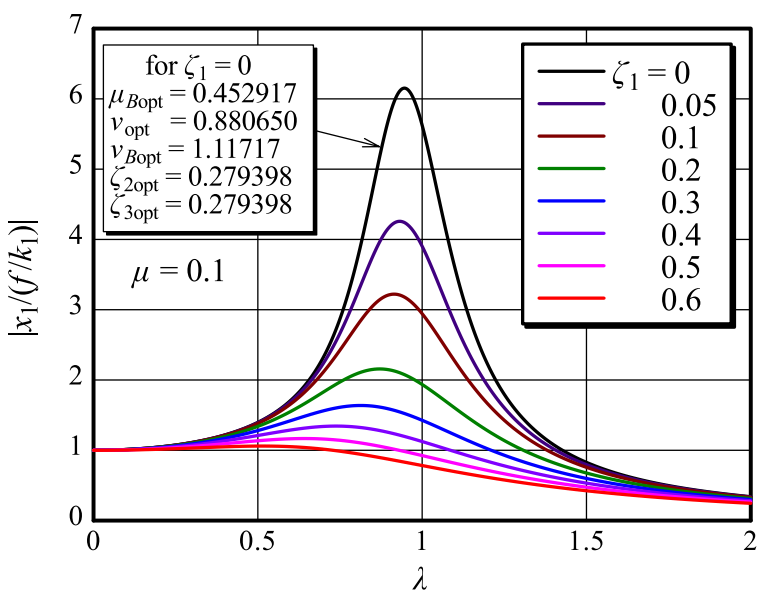

(d) Stability criterion of a parallel-type 3-DOF system $(\mu=0.1)$

Fig. 10 Steady-state responses of a system with the parallel-type double-mass DVA designed by three different optimization criteria. The system with the DVA designed with the stability criterion is characterized by being a multi-degree-of-freedom system with a single resonance point. (d) is the response of a system with a simple design of the DVA with a reduced number of parameters, which is the same as the response (c) of a precisely designed system. 


\section{Discussion}

We now turn to a discussion of the vibration characteristics of the system optimized according to the stability maximization criterion. A comparison of the frequency response of a vibrating system designed with the stability maximization criterion, which aims to attenuate the free vibration in the shortest time, with those of the systems designed with the $H_{\infty}$ and $H_{2}$ optimizations reveals a noticeable shortcoming (see Fig. 10). In this section, we will compare these three optimization criteria for the free-vibration response shown in Fig. 9.

Figure 11 represents the positions of the characteristic roots of a 3-DOF vibratory system optimally designed by the three criteria in the complex plane. Because these characteristic roots are non-dimensionalized, for each resonant frequency, the absolute value of the real part $x$ represents the damping ratio of the system and the imaginary part $y$ represents the ratio of the resonant frequency to the undamped natural angular frequency of the primary system. It can be observed from Fig. 11 that the vibratory systems designed with the $H_{\infty}$ and $H_{2}$ criteria have much smaller values for the damping ratio at each resonant frequency than does the system designed with the stability criterion.

However, in the free-vibration response shown in Fig. 9, the vibration decay of the primary system immediately after excitation is the fastest for the system with the DVA designed based on the $H_{2}$ criterion and the slowest for the system with the DVA designed based on the stability criterion. The reason is that in the vibratory system optimized with the $\mathrm{H}_{2}$ criterion, the kinetic energy applied to the primary system is transferred to the two DVAs. Eventually, at $\tau=15$, the primary mass comes to rest, but then the kinetic energy flows back into the primary system from the DVAs, and the primary mass begins to move again. This exchange of kinetic energy increases the time required for the vibratory system designed based on the $\mathrm{H}_{2}$ criterion to finally come to rest, which can be clearly seen by comparing Fig. 9(c) and (d) at dimensionless time $\tau=50$. The same is true for the system designed according to the $H_{\infty}$ criterion shown in Fig. 9(a), where the primary mass, after once becoming stationary at $\tau=16$, begins to move again. However, in the vibratory system designed according to the stability criterion, the motion of the primary system always precedes that of the DVAs, so that such a reverse flow of kinetic energy does not occur, and the vibrations of the entire system eventually come to rest in the shortest time.

It should be noted that the positions of the characteristic roots from the $H_{\infty}$ and $H_{2}$ solutions depicted in Fig. 11 are all to the right of those of the stability criterion solution. This means that far from the optimal solution, there is no trade-off relationship between the real parts of the three characteristic roots. Figure 12 shows the root trajectories of a vibratory system with a parallel-type double-mass DVA when each of the four design parameters $\left(\mu_{B}, v, v_{B}, \zeta_{2}\right)$ of DVA is varied in the vicinity of the optimal solution. In this figure, the two parameters $\mu$ and $\zeta_{1}$ are fixed at the same constant values, so this is not a complete proof, but at least for this pair of values, we can see that a trade-off relationship is established between the real parts (and between the imaginary parts) of the characteristic roots near the optimal value.

An additional advantage of designing the vibratory system using the stability criterion is that it reduces the load on the DVA springs. It can be seen immediately from Fig. 9 that the displacement of each DVA is kept very small in the vibratory system designed by the stability criterion. This is due to the fact that the optimal damping ratio of each DVA is much larger than those of the other criteria.

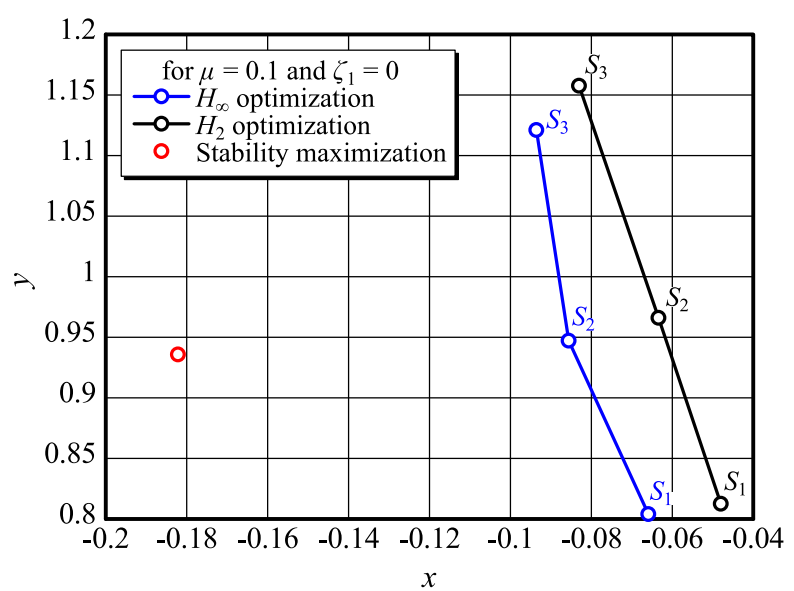

Fig. 11 Coordinates of the characteristic roots of a vibratory system optimized by three different design criteria $\left(H_{\infty}, H_{2}\right.$, and stability criteria). In general, the lower the natural frequency (the smaller the value of $y$ ), the worse the stability of the system (the larger the value of $x$ ). The stability of the vibratory system designed with the stability criterion is much larger than the stabilities of the systems designed with the other two criteria. 


\section{Conclusion}

When we were presenting the optimal solution of a parallel-type double-mass DVA based on the stability criterion in our previous paper, the expressions for calculating the numerical solution were too long to include in full. Therefore, in the present study, we have tried to calculate the optimal solution with simpler formulas while minimizing the degradation of calculation accuracy. As a result of our research, the following conclusions were obtained:

( 1 ) Approximate design formulas (Eqs. (10) and (13)) for the DVA were obtained by equating the damping ratios of two DVAs placed in parallel; in the range of $\mu \leq 0.2$, which is a typical usage condition for DVAs, the stability deterioration rate due to this approximation is less than $0.015 \%$.

( 2 ) The root locus of a system with a single-mass DVA, a system with a series-type double-mass DVA, and a system with a parallel-type double-mass DVA when taking a repeated root was investigated, and the maximum distance from the coordinate origin to the position of the characteristic root was found to be 1 . With the series-type DVA, the system moves while maintaining this maximum value, but in the case of the parallel-type DVA, the system moves closer to the coordinate origin as the mass ratio $\mu$ increases.

( 3 ) The free-vibration response of the system with the double-mass DVA designed by the stability criterion has a slower initial attenuation than the system with the DVA designed by the other optimization criteria, but the final termination of the vibration is the fastest due to the absence of energy backflow from the individual DVAs.

\section{Acknowledgment}

We express our gratitude for being allowed to pursue this research, which was supported by a research grant (19K04276) from the Ministry of Education, Culture, Sports, Science and Technology of Japan.

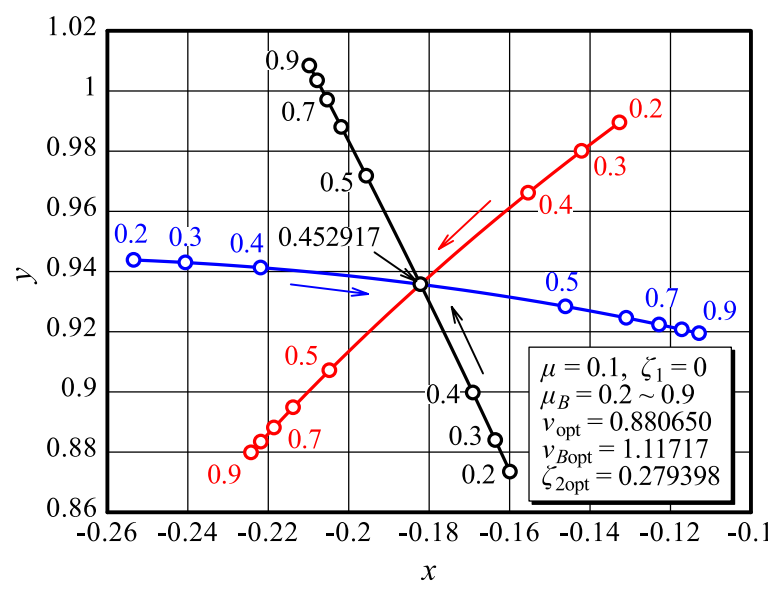

(a) Variation of mass ratio $\mu_{B}$ near the optimal condition

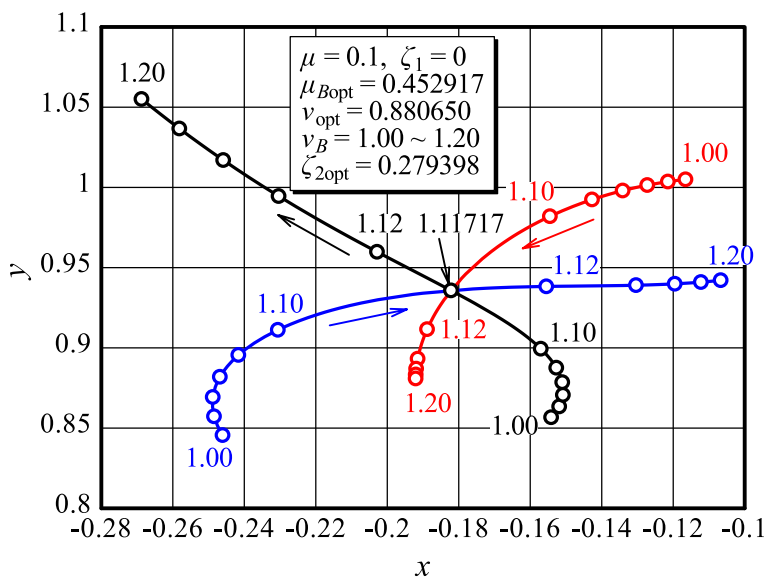

(c) Variation of tuning ratio $v_{B}$ near the optimal condition

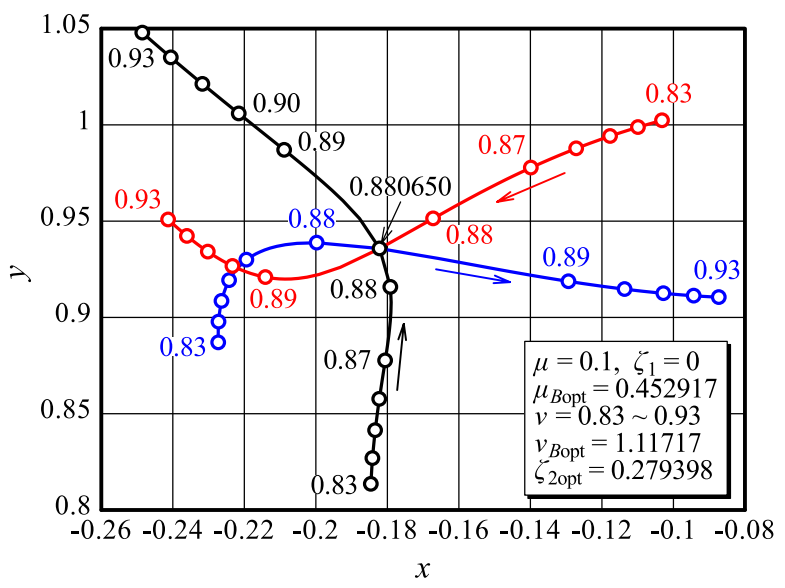

(b) Variation of tuning ratio $v$ near the optimal condition

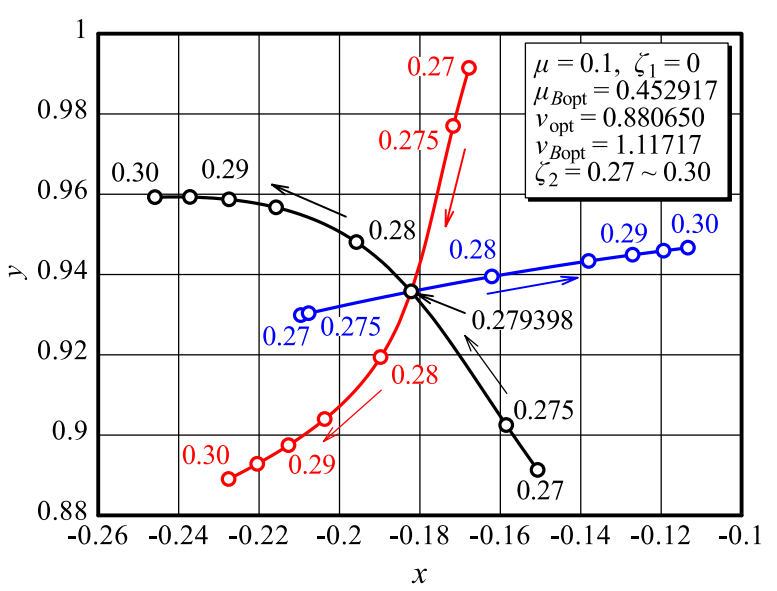

(d) Variation of damping ratio $\zeta_{2}$ near the optimal condition

Fig. 12 Characteristic root trajectories when the value of one design parameter of DVA is varied around its optimal design condition. In the vicinity of the optimal value, there is a trade-off relationship between the real parts of the three characteristic roots, and a change in any one of the four design parameters of DVA will always result in a deterioration of the stability of the system. 


\section{References}

Asami, T., Nishihara, O., and Baz, A. M., Analytical Solutions to $H_{\infty}$ and $H_{2}$ Optimization of Dynamic Vibration Absorbers Attached to Damped Linear Systems, ASME J. Vib. Acoust., Vol.124, No.2 (2002), pp.284-295, DOI: $10.1115 / 1.1456458$

Asami, T., Optimal Design of Double-Mass Dynamic Vibration Absorbers Arranged in Series or in Parallel, ASME J. Vib. Acoust., Vol.139, No.1 (2017), DOI: 10.1115/1.4034776

Asami, T., Mizukawa, Y., and Ise, T., Optimal Design of Double-Mass Dynamic Vibration Absorbers Minimizing the Mobility Transfer Function, ASME J. Vib. Acoust., Vol.140, No.6 (2018), DOI: 10.1115/1.4040229

Asami, T., Exact Algebraic Solution of an Optimal Double-Mass Dynamic Vibration Absorbers Attached to a Damped Primary System, ASME J. Vib. Acoust., Vol.141, No.5 (2019), DOI: 10.1115/1.4043815

Asami, T., and Yamada, K., Numerical Solutions for Optimal Double-Mass Dynamic Vibration Absorbers Attached to a Damped Primary System, Bulletin of the JSME, Mechanical Engineering Journal, Vol.7, No.2 (2020), DOI: 10.1299/mej.19-00051

Asami, T., Calculation of the $H_{\infty}$ Optimized Design of a Single-Mass Dynamic Vibration Absorber Attached to a Damped Primary System, Bulletin of the JSME, Mechanical Engineering Journal, Vol.7, No.5 (2020), DOI: 10.1299/mej.2000250

Brock, J. E., A Note on the Damped Vibration Absorber, ASME Journal of Applied Mechanics, Vol.13, No.4 (1946), p. A-284.

Crandall, S. H., and Mark, W. D., Random Vibration in Mechanical Systems, Academic Press (1963), p.71.

Iwanami, K., and Seto, K., An Optimum Design Method for the Dual Dynamic Damper and Its Effectiveness, Bulletin of the JSME, Vol.27, No.231 (1984), pp.1965-1973.

Kamiya, K., Kamagata, K., Matsumoto, S., and Seto, K., Optimal Design Method for Multi Dynamic Absorber, Transactions of the JSME, Ser. C, Vol.62, No.601 (1996), pp.3400-3405, (in Japanese).

Nishihara, O., and Matsuhisa, H., Design and Tuning of Vibration Control Devices via Stability Criterion, Dynamics and Design Conference '97, No. 97-10-1 (1997), pp.165-168, (in Japanese).

Ormondroyd, J., and Den Hartog, J. P., The Theory of the Dynamic Vibration Absorber, ASME Journal of Applied Mechanics, Vol.50, No.7 (1928), pp.9-22.

Pan, G., and Yasuda, M., Robust Design Method of Multi Dynamic Vibration Absorber, Transactions of the JSME, Ser. C, Vol.71, No.712 (2005), pp. 3430-3436, (in Japanese).

Yamada, K., Free Vibration Analysis of Vibration System with Hysteretic Damping, Dynamics and Design Conference 2020, No.20-11 (2020), p.257(12 pages), (in Japanese).

Yasuda, M., and Pan, G., Optimization of Two-Series-Mass Dynamic Vibration Absorber and Its Vibration Control Performance, Transactions of the JSME, Ser. C, Vol.69, No.688 (2003), pp. 3175-3182, (in Japanese). 